\title{
Perkutane arteriovenöse Shuntanlage bei Patienten mit schwerer COPD: eine neue interventionelle radiologische Technik
}

\author{
Percutaneous Arteriovenous Shunting in Patients with Severe COPD: \\ A New Interventional Radiological Treatment
}

Autoren

Institute
T. Schlosser ${ }^{1}$, M. Burbelko² ${ }^{2}$ M. Ulrich ${ }^{3}$, F. Ludwig ${ }^{4}$, T. Reutiman ${ }^{4}$, G. Antoch ${ }^{5}$, M. Forsting ${ }^{1}$, R. Adamus ${ }^{6}$

Die Institutsangaben sind am Ende des Beitrags gelistet.

Key words
vascular
veins
angiography
interventional procedures
shunts
fistula

eingereicht 10.11 .2011 akzeptiert $\quad 25.10 .2012$

\section{Bibliografie}

Dol http://dx.doi.org/

10.1055/s-0032-1330315

Online-Publikation: 10.12.2012

Fortschr Röntgenstr 2013; 185:

247-252 @ Georg Thieme

Verlag KG Stuttgart · New York . ISSN 1438-9029

\section{Korrespondenzadresse}

\section{Priv.-Doz. Dr.}

\section{Thomas Schlosser}

Institut für Diagnostische und Interventionelle Radiologie und Neuroradiologie,

Universitätsklinikum Essen

Hufelandstraße 55

45122 Essen

Tel.: ++49/2 01/7231501

Fax: ++49/2 01/7231548

Thomas.Schlosser@uni-due.de

\section{Zusammenfassung \\ $\nabla$}

Ziel: Ziel dieser Studie war es, die technische Durchführbarkeit und Sicherheit einer neuen interventionellen radiologischen Technik zur perkutanen Anlage eines arteriovenösen Shunts in der Beckenstrombahn bei Patienten mit schwerer COPD zu evaluieren.

Material und Methoden: 40 Patienten wurden in diese Multicenterstudie eingeschlossen. Bei $38 \mathrm{~Pa}$ tienten konnte die A. iliaca externa erfolgreich mittels neu entwickelter Punktionsnadel von der venösen Beckenstrombahn aus punktiert werden. Durch ein spezielles System wurde ein Nitinol-Implantat (ACS, ROX Medical) zwischen Arterie und Vene eingesetzt, um eine konstante Fistel mit einem Durchmesser von $4 \mathrm{~mm}$ zu gewährleisten.

Ergebnisse: Die Shuntimplantation konnte in 38 Patienten erfolgreich durchgeführt werden. Der perfundierte Shunt konnte mittels DSA gut dokumentiert werden; der Diameter betrug bei allen Patienten zwischen 3 und $4 \mathrm{~mm}$. Periinterventionell trat in einem Patienten eine nicht flusslimitierende Dissektion der A. iliaca externa auf. Postinterventionelle venöse Blutungen im Bereich der Punktionsstelle, die erfolgreich durch lokale Kompression behandelt werden konnten, traten bei 2 Patienten auf. Bei einem Patienten wurde eine periinterventionell aufgetretene periphere arterielle Embolie erfolgreich mittels Lysetherapie behandelt.

Schlussfolgerung: Die in dieser Studie vorgestellte neue interventionelle radiologische Technik zur Implantation eines arteriovenösen Shunts der Beckenstrombahn ist ein sicheres und schnell durchzuführendes Verfahren, das eine niedrige Komplikationsrate aufweist.

\section{Abstract \\ $\nabla$}

Purpose: The aim of this study was to evaluate the technical feasibility and safety of a new interventional radiological technique to create a shunt percutanously between the external iliac vein and artery in patients with severe COPD.

Materials and Methods: 40 patients were included in this multicenter trial. In 38 patients the artery was punctured from the vein using a novel crossing needle. A special delivery system was used to implant a novel nitinol device (ACS, ROX Medical) between the artery and the vein to maintain a $4 \mathrm{~mm}$ calibrated and structured fistula between the two vessels.

Results: Shunt implantation was successful in 38 patients. The perfused arteriovenous shunts could be well documented in DSA and the diameter was measured between 3 and $4 \mathrm{~mm}$ in all cases. Periinterventional non-flow-limiting dissection of the iliac artery occurred in one patient. Post-interventional venous bleeding in two patients was treated successfully by local compression. In one patient a peripheral artery thrombembolism was successfully treated by thrombolysis.

Conclusion: The new interventional radiological technique to create an arteriovenous shunt in the iliac vessels presented in this study has proven to be feasible and safe. 


\section{Einleitung \\ $\nabla$}

Für die Behandlung von Patienten mit schwerer bzw. sehr schwerer chronisch obstruktiver Lungenerkrankung (COPD) gibt es eine Reihe unterschiedlicher Behandlungsstrategien, die vordringlich die medikamentöse Therapie, Atemphysiotherapie, Sauerstofftherapie, bronchoskopische bzw. chirurgische Eingriffe zur Reduktion des Lungenvolumens und die Lungentransplantation umfassen [1]. Trotz dieser unterschiedlichen Therapiekonzepte ist keine dieser Behandlungsoption geeignet, eine langfristige Verschlechterung des Gesundheitszustands aufzuhalten. Die Sauerstofftherapie ist eine der wenigen Behandlungsstrategien, die die Überlebensrate bei Patienten mit COPD und chronischer Hypoxämie verbessert [1 -6]. Ein alternatives Therapiekonzept, das auf eine verbesserte Sauerstoffversorgung abzielt und somit die körperliche Belastbarkeit und Atemnotsymptome verbessern soll, ist die perkutane Anlage eines arteriovenösen Shunts [7, 8]. In dieser Arbeit stellen die Autoren eine neue interventionelle radiologische Technik zur perkutanen Anlage eines arteriovenösen Shunts bei Patienten mit COPD vor, die zum ersten Mal im Rahmen einer klinischen Multicenterstudie Anwendung fand. Die Planung der Studie sowie die klinische Betreuung der Patienten und die Nachkontrollen erfolgten primär durch pneumologische Zentren in Deutschland. Über das klinische Outcome wird an anderer Stelle berichtet.

\section{Prinzip des AV-Shunts}

Ziel des interventionellen radiologischen Eingriffs ist es, einen Shunt zwischen Arterie und Vene in der distalen rechten oder linken Beckenstrombahn (A. iliaca externa und V. iliaca externa) herzustellen. Um eine dauerhafte, in ihrer Größe genau definierte Fistel zu erreichen, wird während der Intervention das von der Firma ROX Medical (San Clemente, CA, USA) entwickelte Anastomotic Coupler System (ACS) implantiert. Aus der Anlage des AVShunts resultierend wird der Sauerstoffgehalt des venösen Blutes im großen Kreislauf erhöht und über einen sauerstoffabhängigen Reflex die pulmonale Durchblutung durch Weitstellung der Lungengefäße vergrößert $[9,10]$. Eine weitere Folge des AV-Shunts resultiert in einer Verminderung des peripheren Gefäßwiderstands, was eine Steigerung des Herzzeitvolumens zur Folge hat [7, 9, 10]. Hierdurch soll eine verbesserte Durchblutung und Sauerstoffabgabe an das Gewebe erzielt werden, mit nachfolgender Steigerung der körperlichen Belastbarkeit [11, 12]. Eine chronische Steigerung des Herzzeitvolumens vergrößert das Schlagvolumen, womit größere Änderungen der Herzzeitvolumina bei Änderungen der Herzfrequenz ermöglicht werden [7]. Ein erhöhtes Herzzeitvolumen aufgrund belastungsinduzierter Tachykardie erlaubt wiederum einen erhöhten Sauerstofftransport und -verbrauch, was für die arbeitende Muskulatur essenziell ist [7]. Das maximale Herzzeitvolumen hängt eng mit dem maximalen Sauerstoffverbrauch zusammen, der wiederum in enger Beziehung zur maximalen Belastbarkeit steht.

\section{Material und Methoden}

$\nabla$

Zwischen Dezember 2008 und November 2010 wurden 40 Patienten (mittleres Alter: 62,2 $\pm 6,6$ Jahre; 18 Frauen, 22 Männer) mit schwerer COPD in die Multicenterstudie eingeschlossen. Demografische und andere Baseline-Charakteristika sind in $\bullet$ Tab. 1 aufgeführt. Prüfplan und Einwilligungserklärung und alle den Studienteilnehmern bereitgestellten Studienmaterialien wurden von der jeweiligen lokalen Ethikkommission genehmigt.

\begin{tabular}{|c|c|}
\hline & $\mathrm{n}=38$ \\
\hline \multicolumn{2}{|l|}{ demografische Charakteristika } \\
\hline Alter (Jahre) & $62,18 \pm 6,66$ \\
\hline männliches Geschlecht; Anzahl (\%) & $21(55,3)$ \\
\hline Body-Mass-Index & $23,51 \pm 4,32$ \\
\hline \multicolumn{2}{|l|}{ Raucheranamnese } \\
\hline - aktuelle oder ehemalige Raucher; Anzahl (\%) & $37(97)$ \\
\hline - Anzahl der pack-years & $39,89 \pm 21,88$ \\
\hline \multicolumn{2}{|l|}{ arterielle Blutgase } \\
\hline $\mathrm{PaO}_{2}(\mathrm{mmHg})(\mathrm{n}=33)$ & $62,37 \pm 8,74$ \\
\hline $\mathrm{PaCO}_{2}(\mathrm{mmHg})(\mathrm{n}=33)$ & $41,61 \pm 5,38$ \\
\hline \multicolumn{2}{|l|}{ FEV $_{1}$ - Post Bronchodilatator } \\
\hline - Grad IV; Anzahl (\%) & $21(55,2)$ \\
\hline - Grad III; Anzahl (\%) & $16(42,1)$ \\
\hline - Grad II; Anzahl (\%) & $1(2,6)$ \\
\hline \multicolumn{2}{|l|}{ Dyspnoe } \\
\hline \multicolumn{2}{|l|}{ NYHA } \\
\hline - Grad 1; Anzahl (\%) & $1(2,6)$ \\
\hline - Grad 2; Anzahl (\%) & $15(39,4)$ \\
\hline - Grad 3; Anzahl (\%) & $21(55,2)$ \\
\hline - Grad 4; Anzahl (\%) & $1(2,6)$ \\
\hline
\end{tabular}

Die Einschlusskriterien waren: eine bekannte COPD im Stadium

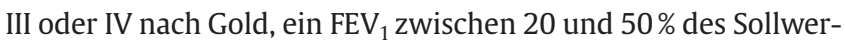
tes, ein BMI $\leq 31,1 \mathrm{~kg} / \mathrm{m}^{2}$ (Männer) oder $\leq 32,3 \mathrm{~kg} / \mathrm{m}^{2}$ (Frauen), abgeschlossene pneumologische Rehabilitation und optimierte medikamentöse Therapie, eine Gehstrecke über $50 \mathrm{~m}$ ohne Sauerstoff im 6-Minuten-Gehtest und eine Verbesserung um 15\% oder $35 \mathrm{~m}$ mit Sauerstoff (oxygen responder), Alter: > 18 Jahre, unterschriebene Einverständniserklärung.

\section{Planungs-CT}

Zur Planung der AV-Shunt-Anlage wurde präinterventionell bei jedem Patienten ein Angio-CT der Beckengefäße durchgeführt. Einerseits dient es vor dem Eingriff dazu, den Verlauf der Beckenarterien und -venen zueinander genau darzustellen, andererseits lassen sich hierdurch verkalkte und nicht verkalkte Plaques der A. iliaca externa, die abhängig vom Grad ihrer Ausbildung und Lokalisation den Eingriff limitieren können, genau darstellen. Voraussetzung für die Durchführbarkeit der AV-Shunt-Anlage ist ein zumindest kurzstreckig paralleler Verlauf von A. und V. iliaca externa. Darüber hinaus müssen die beiden Gefäße in ihrem parallelen Verlauf relativ nah beieinander liegen; der Abstand beider Gefäße sollte $4 \mathrm{~mm}$ nicht übersteigen. Das Anfertigen von MPRs aus den CT-Daten ist für die präinterventionelle Planung und Beurteilung der Gefäßverläufe sehr hilfreich und erlaubt später im Katheterlabor eine gezielte Einstellung der geeigneten Arbeitsprojektionen. Im Bereich der geplanten Fistelanlage sollte außerdem das Vorliegen von verkalkten und nicht verkalkten Plaques ausgeschlossen werden, da hierdurch das periinterventionelle Risiko von z.B. Dissektionen oder peripheren Embolien reduziert werden kann.

\section{Rechtsherzkatheter}

Unmittelbar vor jeder arteriovenösen Shuntanlage wurde ein Rechtsherzkatheter zum Ausschluss einer pulmonalarteriellen Hypertonie durchgeführt. Patienten mit einem mittleren Pulmonalarteriendruck von mehr als $35 \mathrm{mmHg}$ wurden von der Studie ausgeschlossen. 


\section{Anastomotic-Coupler-System (ACS)}

Das Lumen der anastomotischen Verbindung zwischen A. und V. iliaca externa wird durch ein aus Nitinol geformtes Implantat, das ACS, definiert und kalibriert ( $\bullet$ Abb. 1). Das ACS ist auf einem für die perkutane Shuntanlage konzipierten 6F-Implantations-System vormontiert ( $\boldsymbol{O}$ Abb.2). Während des Einsetzens entfalten sich sowohl auf arterieller als auch venöser Seite Stabilisierungsarme, welche so an den Gefäßinnenwänden der Iliacalgefäße anliegen, dass das Implantat eine Seite-zu-Seite-Anastomose mit einem Shuntlumen von $4 \mathrm{~mm}$ Durchmesser definiert.

\section{Shuntanlage}

Zunächst erfolgt die Einlage einer 4-French(F)-Schleuse (1,35 mm) in die A. femoralis comm., über die ein 0,035“-Führungsdraht $(0,89 \mathrm{~mm})$ bzw. alternativ ein speziell für den Eingriff konfigurierter Spiraldraht ( $\bullet$ Abb. 2) bis in die infrarenale Aorta eingeführt

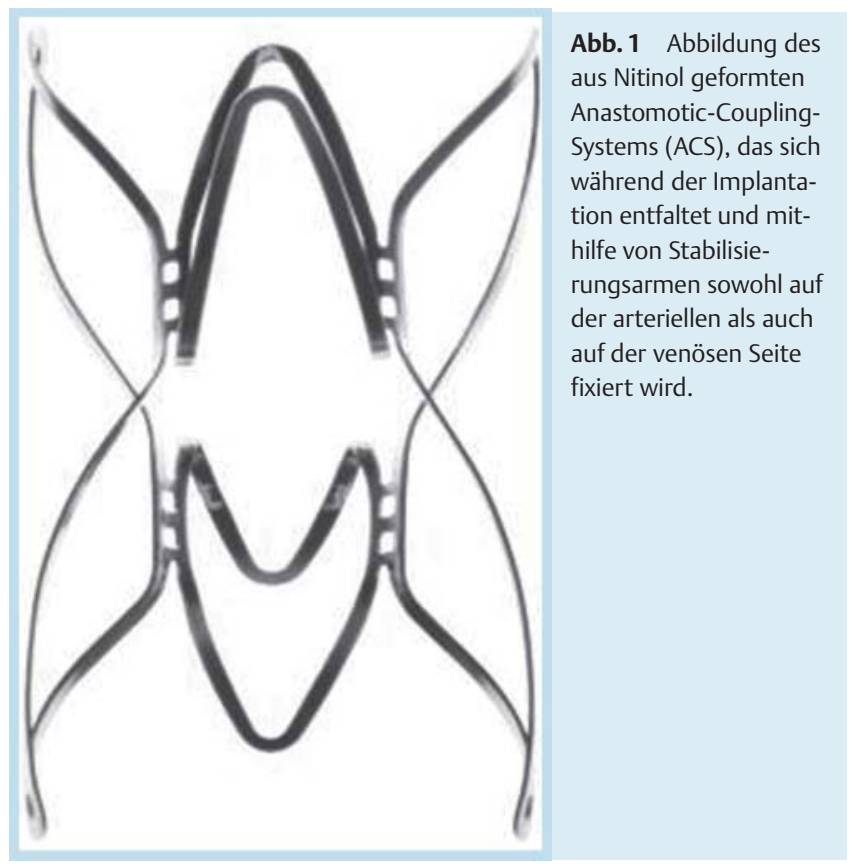

wird. Der Vorteil des Spiraldrahts ist, dass er den Verlauf und die Grenzen des Gefäßlumens der A. iliaca ext. auch ohne Kontrastierung gut darstellt, was später bei der venösarteriellen Punktion sehr hilfreich sein kein. Der Spiraldraht kam erst gegen Ende der Studie bei wenigen Patienten zum Einsatz. Anschließend erfolgt die Punktion der ipsilateralen V. femoralis comm. mit Einlage einer 6F-Schleuse (2,0 mm). Nach Einführen eines 0,035“-Amplatzer-Super-Stiffs oder Backup-Meyer-Drahts über den venösen Zugang und Entfernen der Schleuse wird die venöse Punktionsstelle zunächst mit einem 13F-Dilatator $(4,3 \mathrm{~mm})$ vorgedehnt. Anschließend wird dann vorsichtig eine speziell für den Eingriff konfigurierte, an der Spitze schräg abgeflachte venöse 11F-Schleuse $(3,7 \mathrm{~mm})$ eingeführt ( $\boldsymbol{O} \mathbf{A b b} . \mathbf{2})$, deren intravasale Lage durch eine Kontrastmittelinjektion gesichert werden sollte. Um eine Verletzung der Vene zu vermeiden, die unweigerlich einen Abbruch der Intervention auf der entsprechenden Seite zur Folge hätte, sollte beim Einführen der Schleuse die abgeflachte Seite nach dorsal zeigen. Anhand der aus dem CT bekannten Gefäßverläufe sowie der Drahtpositionen zueinander wird nun eine erste Arbeitsebene eingestellt, bei der Arterie und Vene in überlappender Projektion zur Darstellung kommen ( $\bullet$ Abb.3). Hiervon ausgehend wird die Röntgenröhre nun um $90^{\circ}$ gedreht, um eine parallele Darstellung der Gefäße zu erreichen (Arbeitsebene 2). Durch eine Kontrastmittelinjektion in Arterie und Vene wird nun noch einmal dokumentiert, dass die Distanz zwischen Arterie und Vene nicht mehr als $4 \mathrm{~mm}$ beträgt. Nach Einführen der speziell für den Eingriff entwickelten Punktionsnadel $(\boldsymbol{B}$ Abb. $\mathbf{2}$ ) in die Vene über einen 0,018“Führungsdraht $(0,46 \mathrm{~mm})$ und Zurückschwenken der Röntgenröhre in Arbeitsposition 1 erfolgt nun das „Crossing-Manöver“, also die Punktion der Arterie von der Vene aus. Hierbei wird die gebogene Punktionsnadel an der geeigneten Stelle positioniert, durch leichte Rotationsbewegungen die optimale Ausrichtung der Nadelspitze in Richtung der Arterie ertastet und durch Vorschub der Nadel aus der Schutzhülle die Venen- und Arterienwand durchstoßen. Eine korrekte Lage der Punktionsnadel im Lumen der Aorta wird durch Drahtvorschub dokumentiert. Nach Entfernen der Punktionsnadel und Belassen des Führungsdrahts in Vene und Arterie wird erneut Arbeitsebene 2 eingestellt und durch eine Kontrastmittelinjektion in beide Gefäße sichergestellt, dass kein Auseinanderweichen der Gefäße durch ein zwischenzeitlich auf-

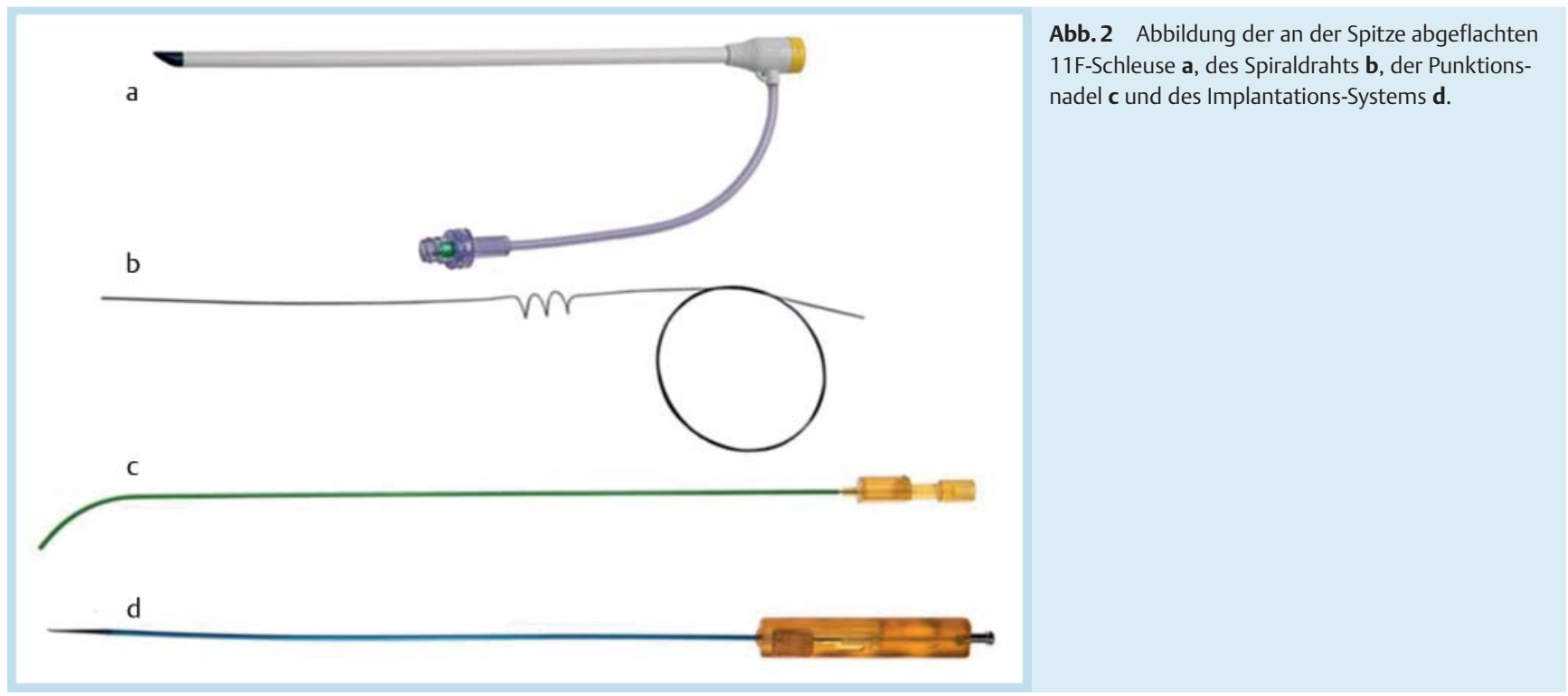



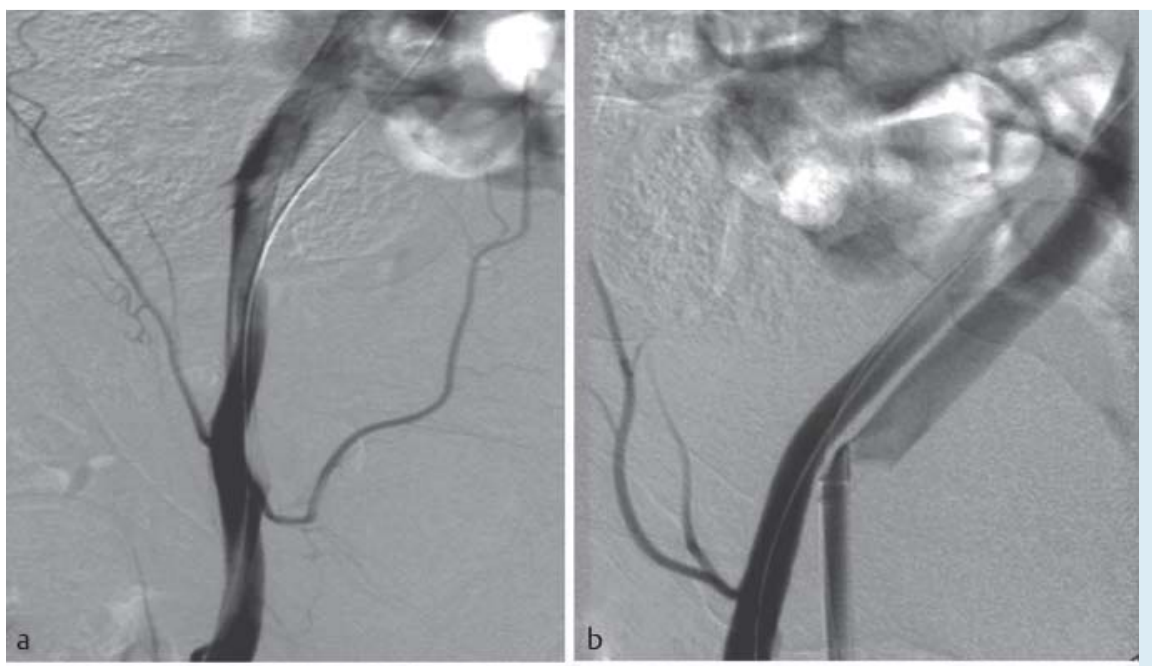

Abb.3 a Arbeitsprojektion A mit überlappender Darstellung der kontrastierten rechten $A$. iliaca externa und der durch einen Führungsdraht markierten V. iliaca externa. b Arbeitsprojektion B mit Darstellung der parallel verlaufenden, kontrastierten Beckengefäße nach venös-arterieller Punktion und über dem Implantationspunkt einliegenden Führungsdraht in Vene und Arterie. Der Implantationspunkt befindet sich $\mathrm{ca} .3 \mathrm{~cm}$ proximal des $\mathrm{Ab}$ gangs der A. circumflexa ilium profunda. $\mathbf{c}$ Kontrastierung der Vene und Arterie nach erfolgreicher Implantation des ACS zum Ausschluss eines Hämatoms und Dokumentation einer regelrechten Implantatlage. d Nach arterieller Kontrastinjektion zeigt sich eine deutliche AV-Fistel mit Jetphänomen und Kontrastierung der V. iliaca externa.
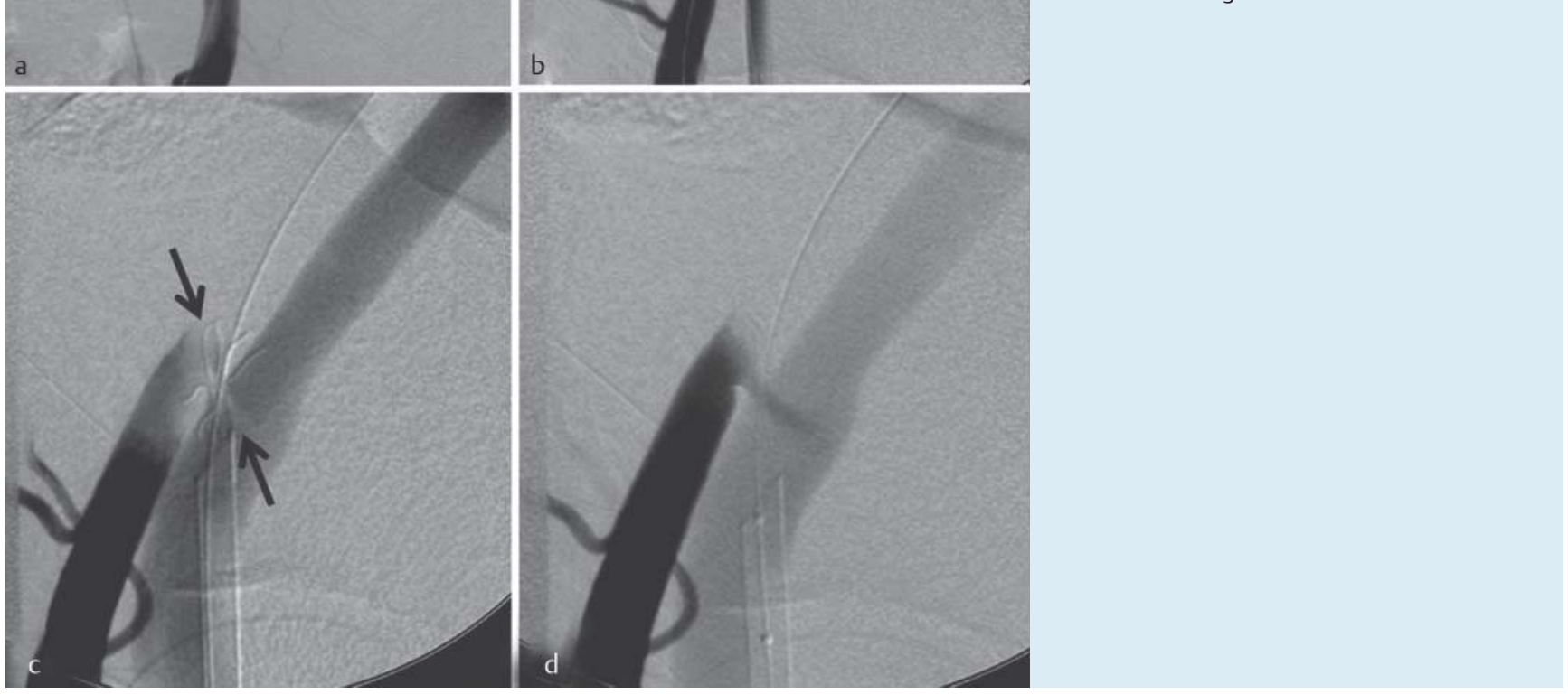

getretenes Hämatom stattgefunden hat ( $\bullet$ Abb.3). Über den weiterhin einliegenden 0,018“-Führungsdraht erfolgt nun die Implantation des ACS ( $\bullet$ Abb. 1), das in die Seit-zu-Seit-Anastomose zwischen A. und V. iliaca externa implantiert wird. Hierzu wird nun das an der Spitze konisch konfigurierte Implantations-System ( $\bullet$ Abb.2) über den 0,018“-Führungsdraht von der Vene in die Arterie eingeführt und durch Zurückziehen der Schutzhülle, das in zwei Schritten durchzuführen ist, das ACS je zur Hälfte in der Arterie und Vene verankert. Bei der Freisetzung des ACS wird die $11 \mathrm{~F}-\mathrm{Schleuse}$ an den Implantationspunkt vorgeschoben und durch leichten Druck auf die Schleuse ein Auseinanderweichen beider Gefäße verhindert. Nach arterieller Kontrastinjektion zur Kontrolle einer regelrechten ACS-Implantation und Fistelanlage erfolgt als letzter Schritt die Dilatation der Fistel mittels eines $4 \mathrm{~mm}$-Ballonkatheters, die hinterher erneut durch eine Kontrastinjektion dokumentiert werden sollte ( $\bullet$ Abb.3). Der Shuntdurchmesser wurde in unserer Studie anhand der kalibrierten DSA-Bilder bestimmt. Die Kalibrierung wurde mittels der Ballonmarker durchgeführt. Die meisten Patienten wurden während der Intervention nicht speziell antikoaguliert, wohingegen bei allen Patienten nach der AV-Shuntanlage eine Thrombozytenaggregationshemmung mit $100 \mathrm{mg}$ ASS für ein Jahr durchgeführt wurde.

Da die Implantation des ACS und die nachfolgende Ballondilatation mit Schmerzen für den Patienten verbunden sein kann, sollte zu Beginn der Intervention eine Analgesie mit Piritramid (Dipidolor 7,5 mg i. v.) oder Pethidin (Dolantin 50 mg i. v.) als Kurzinfu- sion durchgeführt werden. Nach Entfernung der Katheter und Schleusen erfolgen die manuelle Kompression der Punktionsstellen und die Anlage eines Druckverbands mit nachfolgender Bettruhe für $24 \mathrm{~h}$ und Low-dose-Heparinisierung.

\section{Ergebnisse \\ $\nabla$}

In 38 von 40 Patienten (95\%) konnte die AV-Shuntanlage erfolgreich durchgeführt werden. Bei diesen Patienten betrug die Fistelgröße zwischen 3 und $4 \mathrm{~mm}$. Die durchschnittliche Interventionsdauer betrug $43 \pm 12 \mathrm{~min}$. Bei einem Patienten wurde aufgrund ausgeprägter verkalkter und nicht verkalkter Plaques in der arteriellen Beckenstrombahn auf die Implantation des ACS verzichtet. Bei einem weiteren Patienten konnte die venösarterielle Punktion aufgrund ungeeigneter Gefäßverläufe nicht erfolgreich durchgeführt werden. Bei zwei Patienten wurden nach Einlage der venösen 11F-Schleuse geringe Kontrastmittelparavasate nachgewiesen. In einem Fall wurde daraufhin die Intervention auf der kontralateralen Seite erfolgreich durchgeführt. In dem zweiten Fall erfolgte die Shuntanlage ipsilateral nach 4 Wochen. Bei zwei Patienten traten postinterventionell relevante Hämatome im Bereich der venösen Punktionsstelle und im proximalen Oberschenkel auf, die jedoch erfolgreich konservativ behandelt werden konnten. In einem Fall wurde nach der venösarteriellen Punktion eine kurzstreckige arterielle Dissektion be- 
obachtet, die jedoch nicht flusslimitierend war und keine Limitation für die Implantation des ACS darstellte. Ein umschriebener venöser Thrombus zeigte sich bei einem Patienten im Bereich des implantierten ACS, der jedoch nach Gabe von 5000i. E. Heparin nicht mehr nachweisbar war. In einem Fall trat nach Implantation des ACS eine periphere Embolie mit Verschluss der A. tibialis anterior auf, welcher am Folgetag durch eine 24-stündige Lysetherapie erfolgreich und ohne Komplikationen rekanalisiert werden konnte.

\section{Diskussion}

Die chronisch obstruktive Lungenerkrankung ist eine progressive Erkrankung, die die Kombination aus chronischer Bronchitis und Lungenemphysem beinhaltet $[13,14]$. Die COPD ist die häufigste chronische Lungenerkrankung; allein in Deutschland sind zwischen 3 und 5 Millionen Menschen an einer COPD erkrankt, wobei schätzungsweise fast $1 \%$ der Bevölkerung unter schwerer bzw. schwerster COPD im Stadium III bzw. IV leidet. Eine Alternative zu den etablierten Behandlungsstrategien zur Verbesserung der Atemnot und körperlichen Belastbarkeit durch Erhöhung der gemischtvenösen Oxygenierung ist die in dieser Arbeit vorgestellte neue interventionelle-radiologische Technik zur Anlage einer arteriovenösen Fistel.

In der vorliegenden Studie konnte das ACS in 95\% (38 von 40) der Fälle erfolgreich implantiert werden. In sämtlichen dieser Fälle zeigte sich nach der Implantation ein arteriovenöser Shunt mit einem Fisteldurchmesser von 3-4 mm. In keinem der Fälle trat eine schwerwiegende Komplikation in Form einer retroperitonealen Blutung auf, wodurch die Sicherheit der Implantationstechnik dokumentiert wird. In zwei Fällen traten nach Entfernung der Schleusen relevante Hämatome im Bereich der venösen Punktionsstelle auf, die konservativ behandelt werden konnten. In beiden Fällen lag eine relativ tiefe Punktion der V. femoralis communis vor, verbunden mit einer perinterventionellen Gabe von $500 \mathrm{mg}$ Aspirin i.v. sowie 5000i. E. Heparin i.v., was in Kombination mit dem Entfernen relativ großlumiger venöser Schleusen und der venösen Druckerhöhung nach Fistelanlage als Ursache für die Blutungen angesehen werden kann. In weiteren Fällen wurde auf die perinterventionelle Antikoagulation und Gabe von Thrombozytenaggregationshemmern verzichtet; es wurde lediglich eine Low-dose-Heparinisierung für $24 \mathrm{~h}$ durchgeführt. Bei einem Patienten trat unmittelbar nach der venösarteriellen Punktion eine kurzstreckige arterielle Dissektion im Bereich des Implantationspunkts auf, die jedoch weder flusslimitierend war noch als Limitation für die ACS-Implantation angesehen wurde. Auch hier konnte die AV-Fistel regelrecht implantiert werden. Der Patient zeigte im CT relativ deutliche nicht verkalkte Plaques im Bereich der arteriellen Beckenstrombahn, was die Bedeutung einer sorgfältigen Patientenselektion im Vorfeld des Eingriffs unterstreicht. In einem weiteren Fall trat während der Intervention eine periphere Embolie mit Verschluss der A. tibialis anterior auf. Durch 24-stündige Lysetherapie konnte das Gefäß wieder erfolgreich rekanalisiert werden. Als Ursache für die periphere Embolie wird in diesem Fall eine iatrogene Plaqueablösung angesehen, die möglichweise durch Manipulation des Spiraldrahts oder während der venösarteriellen Punktion hervorgerufen wurde. Auch hier ist anzunehmen, dass das Risiko für derartige Komplikationen mit dem Ausmaß der Plaquelast steigt. Eine venöse Thrombenbildung im Bereich des ACS trat in nur einem Fall auf und konnte durch einmalige Gabe von 5000i. E. Heparin erfolgreich therapiert werden.

Einfluss der AV-Fistel auf den pulmonalarteriellen Druck Patienten mit COPD können aufgrund des erhöhten Afterloads, der durch ein vaskuläres Remodeling oder durch hypoxische Vasokonstriktion zustande kommt, eine Rechtsherzinsuffizienz entwickeln. Ein durch eine AV-Fistel hervorgerufenes erhöhtes HZV kann prinzipiell zu einer weiteren Erhöhung des pulmonalarteriellen Druckes führen. Wenn jedoch eine Erhöhung der gemischtvenösen Sauerstoffsättigung mit einer Verbesserung der Oxygenierung in der Lunge einhergeht, kann dies zu einer Reduktion der hypoxischen pulmonalen Vasokonstriktion, des pulmonalen Gefäßwiderstands und des pulmonalarteriellen Druckes führen [14]. Dass diese Effekte tatsächlich auftreten, konnte in einer Studie von Faul et al. gezeigt werden [8], in der bei 12 Patienten mit schwerer COPD mittels operativer bzw. perkutaner Vorgehensweise eine AV-Fistel angelegt wurde. Nach 12 Wochen konnte hier eine nummerische, wenn auch nicht statistisch signifikante Senkung des pulmonalarteriellen Druckes von 29,1 auf $26,3 \mathrm{mmHg}$ beobachtet werden. Die 6-min-Gehstrecke konnte nach 12 Wochen im Mittel von $217 \mathrm{~m}$ auf $276 \mathrm{~m}$ gesteigert werden. Dieser Anstieg war statistisch signifikant. In einer weiteren, an 15 Patienten durchgeführten Studie zeigte sich nach perkutaner AV-Shunt-Anlage eine signifikante Steigerung des Herzminutenvolumens und des Sauerstoffangebots, jedoch bestand ein Trend zu einer Reduktion der 6-min-Gehstrecke [15].

\section{Schlussfolgerung}

Die perkutane arteriovenöse Shuntanlage mit Implantation des ACS der Firma ROX Medical bei Patienten mit schwerer COPD ist ein sicheres und schnell durchzuführendes Verfahren, das eine niedrige Komplikationsrate aufweist. Die in unserer Multicenterstudie aufgetretenen periinterventionellen Komplikationen waren allesamt beherrschbar. Schwerwiegende Komplikationen in Form von retroperitonealen Blutungen traten nicht auf. Weitere Studien und Nachuntersuchungen sollen eine verbesserte Oxygenierung bei den Patienten mit schwerer COPD, die Langzeitoffenheitsrate des ACS sowie den klinischen Nutzen der Methode untersuchen und dokumentieren.

\footnotetext{
Institute

1 Institut für Diagnostische und Interventionelle Radiologie und Neuroradiologie, Universitätsklinikum Essen

2 Klinik für Strahlendiagnostik, Philipps-Universität, Marburg

3 Klinik für Innere Medizin I/Angiologie/Kardiologie, Parkkrankenhaus Leipzig

4 ROX Medical, San Clemente, CA, USA

5 Institut für Diagnostische und Interventionelle Radiologie, Universitätsklinikum Düsseldorf

6 Institut für Radiologie, Klinikum Nürnberg Nord
}

\section{Literatur}

1 Pauwels RA, Buist AS, Calverley PM et al. Global strategy for the diagnosis, management, and prevention of chronic obstructive pulmonary disease. NHLBI/WHO Global Initiative for Chronic Obstructive Lung Disease (GOLD) Workshop summary. Am J Respir Crit Care Med 2001; 163: $1256-1276$

2 Nocturnal Oxygen Therapy Trial Group. Continuous or nocturnal oxygen therapy in hypoxemic chronic obstructive lung disease: a clinical trial. Ann Intern Med 1980; 93: 391 - 398

3 Long term domiciliary oxygen therapy in chronic hypoxic cor pulmonale complicating chronic bronchitis and emphysema. Report of the Medical Research Council Working Party. Lancet 1981; 1: 681-686

4 Flenley DC. Long-term home oxygen therapy. Chest 1985; 87: 99-103 
5 Stoller JK, Panos RJ, Krachman S et al. Oxygen therapy for patients with COPD: current evidence and the long-term oxygen treatment trial. Chest 2010; 138: 179-187

6 Doherty DE, Petty TL, Bailey W et al. Recommendations of the 6th longterm oxygen therapy consensus conference. Respir Care 2006; 51: 519-525

7 Faul JL, Sievert H. Percutaneous Creation of Arteriovenous Shunts. Vascular Disease Management 2008; 5: 128-133

8 Faul JL, Galindo J, Posadas-Valay $R$ et al. An arteriovenous fistula increases exercise capacity in patients with COPD. Chest 2010; 138 : $52-58$

9 Sandoval J, Lopez R, Beltran U et al. Effect of hydralazine on intrapulmonary shunt. Crit Care Med 1986; 14: 689-692

10 Schumacker PT, Newell JC, Saba TM et al. Ventilation-perfusion relationships with high cardiac output in lobar atelectasis. J Appl Physiol 1981; 50: $341-347$
11 Kelly PJ. Oxygen saturation, Po2, Pco2, and pH in tibial bone blood distal to femoral arteriovenous fistula in puppies. J Lab Clin Med 1969; 73: $418-424$

12 Nishimura T, Faul JL, Berry GJ et al. Effect of a surgical aortocaval fistula on monocrotaline-induced pulmonary hypertension. Crit Care Med 2003; 31: $1213-1218$

13 Fabbri LM, Hurd SS. Global Strategy for the Diagnosis, Management and Prevention of COPD: 2003 update. Eur Respir J 2003; 22: 1-2

14 Cooper CB, Celli B. Venous admixture in COPD: pathophysiology and therapeutic approaches. Copd 2008; 5: 376-381

15 Bertog SC, Kolmer C, Kleschnew S et al. Percutaneous femoral arteriovenous shunt creation for advanced chronic obstructive pulmonary disease: a single-center safety and efficacy study. Circ Cardiovasc Interv 2012; 5: 118-26 